\title{
Design a Radon Exposure Technique for Risks Evaluation of Radon's Progenies Accumulation on the Platelet and Red Blood Cells
}

\author{
Asaad H. Ismail and Mohamad S. Jaafar
}

\begin{abstract}
Risks evaluation of alpha particles deposition on human blood has been performed by using new exposure techniques of CR-39 NTDs. The method of distribution of alpha particles and radiation dose on the surface of CR-39 NTDs showed that human blood samples have significantly improved. Platelet (PLT), red blood cells (RBC) and white blood cells (WBC) counts were found to be relatively affected. However, the most was the PLT count, which decreased with increasing radiation dose of alpha particles. In addition, comparative study of CR-39 NTDs and the human blood samples is a new technique for in vitro studies of blood ionization. In the present exposure technique, the radon concentration reduced to around $4.915 \%$, and thus, around $95 \%$ of the radon concentration was saved. A ratio of the deposition of alpha particles was found to be adequate to change the PLT count in both males and females.
\end{abstract}

Index Terms-Human blood, CR-39 NTDs, Radon-222, Alpha particles.

\section{INTRODUCTION}

Hematology studies in the field of radiation have an active role in estimating the increases in the number of chromosomal aberrations in human blood lymphocytes due to exposure to ionization radiation. This increase mainly reflects the last year of exposure, owing to repair mechanism, and limit the usefulness of this parameter as a marker of long-term exposure [1]. Exposure to ionizing radiation higher than environmental radiation can result in different diseases, especially blood cancer. Leukemia results from infection in the blood components. High amount of white blood cells can be a sign of infection, and increases in certain types of leukemia. Low white blood cell counts can be a sign of bone marrow diseases or an enlarged spleen. High level of hemoglobin $(\mathrm{Hgb})$ can occur due to lung disease, living at high altitude, or excessive bone marrow production of blood cells. Decrease in the number of platelets in the blood due to thrombocytopenia can result in poor blood clotting[1-2].

Whenever an alpha particle (which are emitting from radon's progenies) passes through a cell nucleus, the DNA is likely to be damaged (ionized), because the penetrated alpha particle may cause genomic changes typically in the form of point mutations and transformations. Alpha particles are more damaging to the living tissue, because they are more massive and more highly charged than other types of ionizing

Manuscript received May 15, 2011; revised June 3, 2011. This work was supported in part by the USM Grant No. PFIZIK/843099.

A.H.Ismail is with the Universiti Sains Malaysia, 11800 USM, Penang, Malaysia (telephone: 006-196890766, e-mail:asadhawlery@hotmail.com).

M. S. Jafaar is with the Universiti Sains Malaysia, 11800 USM, Penang, Malaysia (telephone: 006-194785418, e-mail: msj@usm.my). radiation [3-4].

The effective grade of the ionization of incident alpha particles depends on its energy and time of exposure, and can cause an excitation of molecules in blood, resulting in their vibration and ionization. An immediate decrease in the lymphocytes may not spontaneously affect the other mature blood cells. However, all blood cells are constantly replaced by new ones. If these replacements fail because of the damage to the bone marrow, the population of white blood cells will drop in a few days after exposure, sometimes followed by a rather slow decrease in the red blood cells. Red blood cells live longer than white blood cells, and they are replaced more gradually, and thus the effect is seen somewhat later[1,5].

This research includes a fabrication of new exposure technique of human blood samples to a known radon concentration using CR-39 NTDs as a main detector. This technique consisted of counting the radiation damage track that is produced by alpha particles in a certain plastic process, and evaluate the deposition of alpha particles on the human blood disease. The objective of this study is to evaluate the usefulness of platelet counts at the time of diagnosis as a prognostic factor, and to estimate the optimum dose/time of irradiation for in vitro study on blood parameter compliments. Low radiation dose of radium $\left({ }^{226} \mathrm{Ra}: 5 \mu \mathrm{Ci}\right)$ is used to study the hematological effects of low radiation with CR-39 NTDs techniques. Complete blood count (CBC) has been calculated for the blood samples before, after irradiation, and at different doses and irradiation times.

\section{RESEARCH METHODOLOGY}

The procedure of fabricating a radon exposure technique consists of the following stages:

a) Preparation of a chamber of radon gas (source of radon gas): one of the PVC cylinder chambers (length $=21 \mathrm{~cm}$; diameter $=7 \mathrm{~cm}$ ) equipped with the two pips has been used for a combination of radon gas, as shown in Fig.1A.

b) Two sources of radium $\left({ }^{226} \mathrm{Ra}=5 \mu \mathrm{Ci}\right)$ stacked in safely (on the base of the PVC chamber) inside the cylinder's plastic chamber, as shown in Fig.1B. In addition, an electric fan has been supported inside the PVC chamber (stacked on top -ceiling), as shown in Fig.1C to circulate radon gas within the exposure process. The radium source and the chamber of radon gas are stored inside the lead box, as shown in Fig.1D.

c) The chamber is left as it is until the gas reaches saturation; it was $50.1 \mathrm{kBq} / \mathrm{m}^{3}$ in around 28 days. RAD 7 was used to measure radon concentration inside that chamber. 

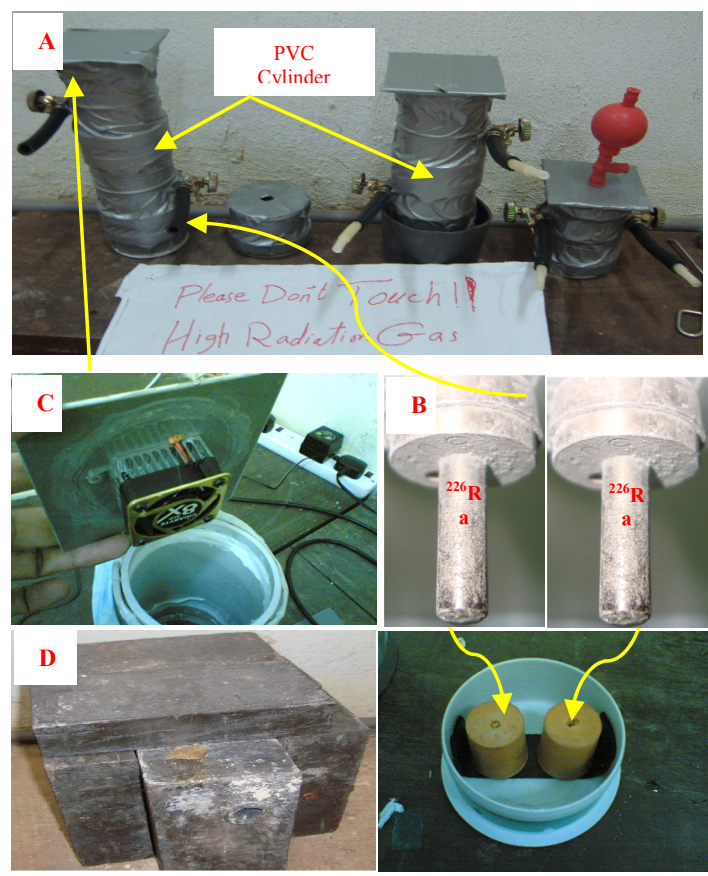

Fig.1: Tools and stages of preparing a chamber of radon gas

d) Preparing private dimension of the blood dispenser, the internal dish for the cover head of the mineral water found has a suitable dimension to doing dishes to put blood samples in it, as shown in Fig.2. Blood samples were prepared inside a plastic dish (diameter $=1.42 \mathrm{~cm}$ : depth $=0.5 \mathrm{~cm})$. Therefore, depth of the plastic dish $(=0.5 \mathrm{~cm})$, blood volume $(=0.5 \mathrm{ml})$ and place of blood samples were justified with the thickness and the place of CR-39 NTDs. Thus, the number of monuments belonging to the surface of the detector was utilized to calculate the amount of alpha particle incidents on the human blood samples.

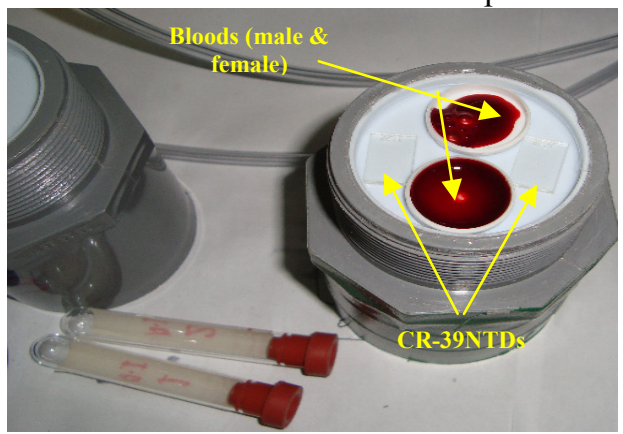

Fig.2: Human blood exposure to radon gas.

e) Radiation dosimeter-type VICTOREEN was used to measure the average dose inside the radon dosimeter (PVC; radius $=6 \mathrm{~cm} \&$ length $=7 \mathrm{~cm}$,) as shown in Fig. 3. RAD7 was used to measure the radon concentration in each stage.

f) Alpha track density into the surface of CR-39 NTDs was utilized to evaluate alpha track density in the surface of the human blood samples. Thus, the concept of the present design was to expos human blood samples with radon's progeny that came from radiation effects on blood components via evaluating alpha particle density, range, and restricted energy loss of it into the blood.

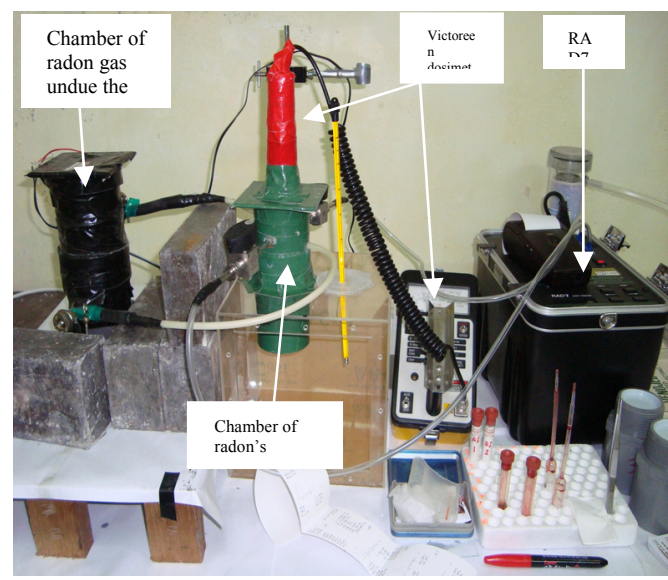

Fig.3: Installed of the radon exposure technique

g) Specifically, Rad7 was used to demonstrate that the CR-39 NTDs are in high efficiency. Continuity in the measurement of exposure and declined in further comments are two ways; RAD7 and CR-39 NTDs are considered important points in this technique. The schema diagram is displayed in Fig.4.

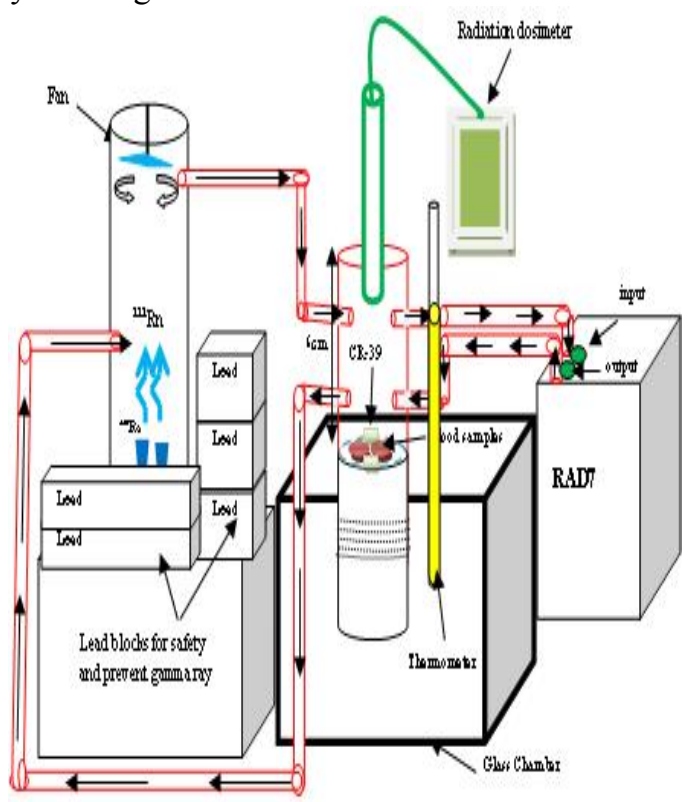

Fig.4: Schema diagram of human blood exposed in vitro to radon gas (irradiation technique).

h) In a primary test, the effects of transfusion of the blood samples from withdrawing place (Clinical of USM/Main campus) to the place of exposure (Biophysics lab / School of Physics) on the components (WEB, RBC and PLT) of the human blood samples has been done for 20 minutes (maximum time of blood exposure for the case state) without exposure to radon gas (except the background $=204 \pm 72 \mathrm{~Bq} / \mathrm{m}^{3}$ ).

i) Both blood samples and CR-39 NTDs moved slowly without any effect on radon concentration. Thus, blood samples will be mixable, and that to avoid deposition of a plasma above red blood. The process of the exposure was in several times ( $=6,10,15$, and 20 minutes). Age of the blood samples was not taken into account (29 and 30 years old for male and female), blood tests before and after irradiation was less than one hour.

The average radiation dose rate inside a chamber of radon gas was $41 \pm 1.5 \mu \mathrm{Sv} / \mathrm{h}$, and radiation dosimeter (Victoreen) has been used to measure the mentioned does. On 
the other hand, the average dose rate inside an exposure chamber was $7.7 \pm 0.041 \mu \mathrm{Sv} / \mathrm{h}$. Whole blood samples were transported at a temperature of $\left(27 \pm 2.2 \mathrm{C}^{0}\right)$ inside the technique. SRIM 2008 program [6] was used to calculate the range of alpha particle and restrict energy loss into the blood and the CR-39 NTDs, as shown in Fig.5.
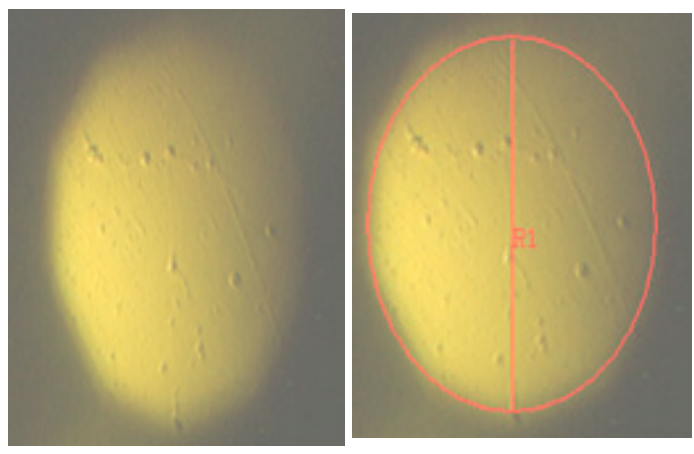

Fig.5: Alpha track register by CR-39 NTDs (Area $0.101912 \mathrm{~mm}^{2}$ )

\section{RESUlTS AND DisCUSSION}

The present exposure technique had a little percentage of loss of radon concentration in each stage of exposure, as shown in Fig.6. Because there was an absorbed dose by CR-39NTDs and the blood sample, the mentioned percentage loss has not been considered as a bad point for the present technique. In addition, the process of mixing blood samples affected the maintenance of radon concentration. This relatively is an effect on the efficiency of present technique

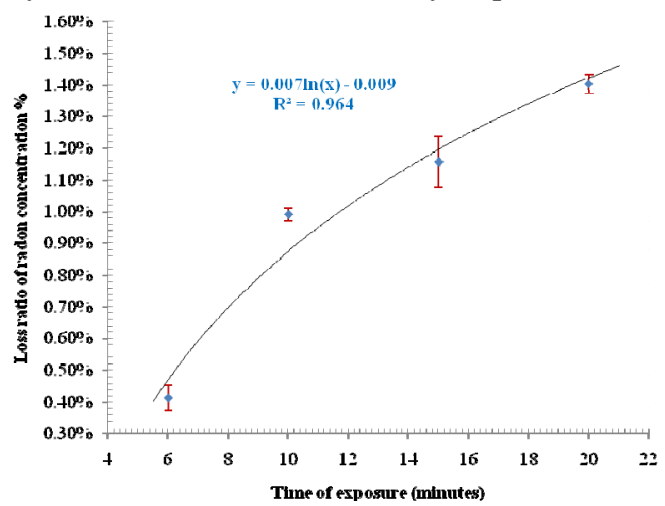

Fig.6: Percentage decreases of radon concentration with the stages of exposure during exposure.

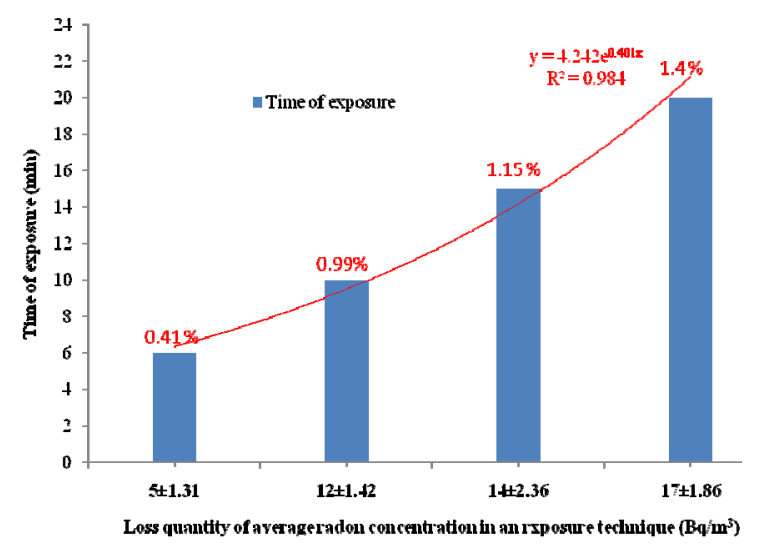

Fig.7: Average loss ratio of radon concentration depends on time of exposure

Average loss ratio of radon concentration varied from $0.413 \%$ to $1.404 \%$; this depends on the time of exposure, as shown in Fig.7. Average value of radon concentration was $2198 \pm 5.099 \mathrm{~Bq} / \mathrm{m}^{3}$. Radon concentration inside the chamber of exposure not reduced rapidly, as shown in Fig. 8 .

Radon concentration was not constant for the exposure stages; there was a percentage loss of its value. This was because in each stage of exposure, and after closing Pip steel, the low value of the gas will depend on when the PVC chamber is open to the next stage. In addition, the rate of radium decay inside the big chamber to produce radon gas for that time is not enough to substitute (compensation) the rate loss of radon gas. Therefore, there is no ideal technique to exposure without losing a few of its quantity.

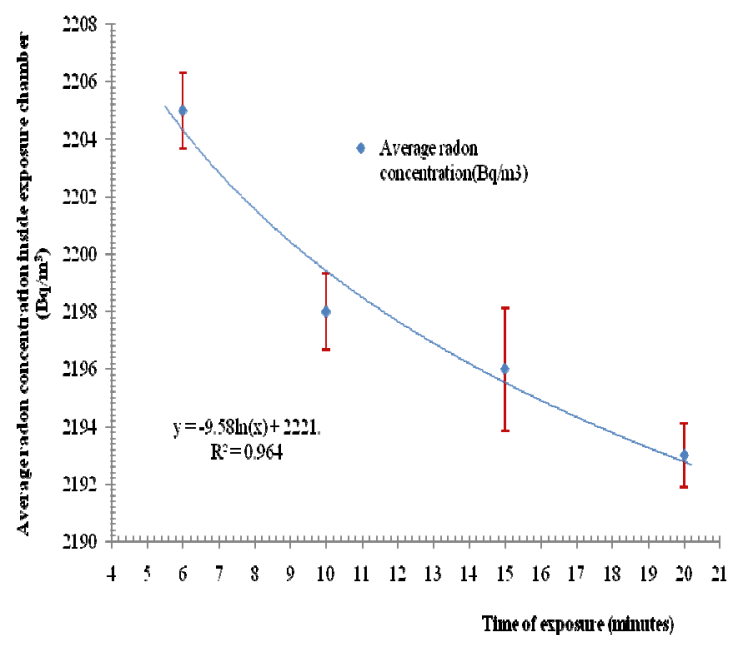

Fig.8: Changes of radon concentration with the time of exposure

The average dose rate inside the radon exposure chamber that was used to expos blood and CR-39 NTDs varied, and depended on the time of exposure, as shown in Fig.9.

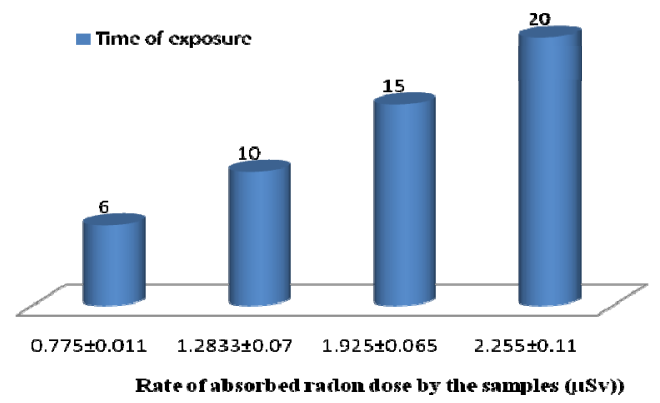

Fig.9: Rate and percentage occupation of absorption dose into blood samples for various exposure times

Pursuant to the time of exposure of the radon concentration to that of selected samples (CR-39 NTDs +0.5 $\mathrm{ml}$ of blood sample), the density of the deposition of alpha particles in the selected samples was varied, as shown in Fig. 10.

Depositions of the ratio of alpha particles as mentioned in Fig. 11 We were relatively sufficient to make a change in platelet blood count and RBC for both males and females, and it was not sufficient to make changes in WBC. Platelets count for the male was more affected than the female. This is clear from the statistical analysis of the Pearson correction of male and female $(=0.924 ; \mathrm{P}-$ Value $=0.076)$. The reason could be that each liter of the male blood contains about 150-160 grams of hemoglobin, compared to only 130-140 grams for females. The bottom line is that each liter of male blood can 
carry about 11 per cent more oxygen than a similar quantity of female blood [7]. As well as, producing free radicals will be more accurate at the blood of male than the female. The decrease of PLT count is not correlated with the absorbed dose in Fig. 11.

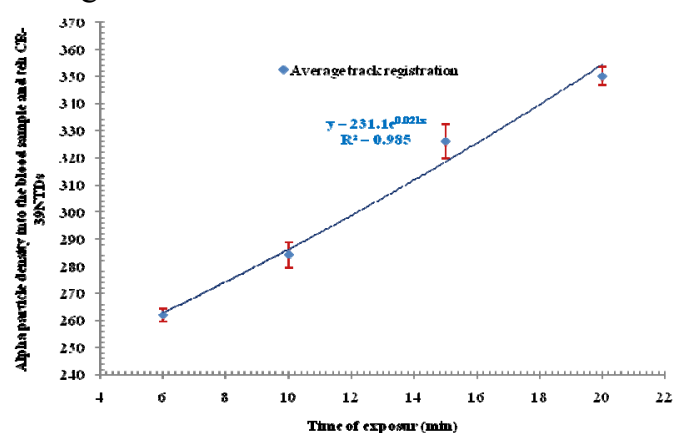

Fig.10: Increasing the deposition of alpha particles with the time of exposure

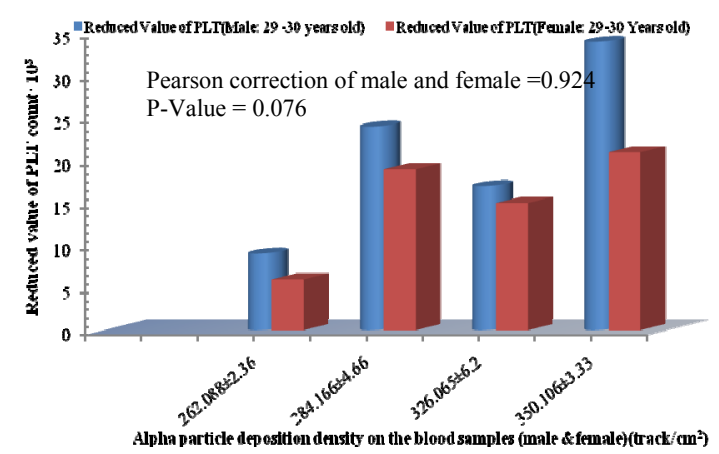

Fig.11: Relative changes in reduced of platelet count (PLT) with rate of alpha particle deposition on the blood

This is due to the energy of alpha particles, which are emitted from the radon's daughters and deposited onto the samples did not have same energy. Accordingly, the penetration depth of the alpha particles will be a difference, and then the rate of ionization will be not uniform. Therefore, the distribution of the ionized particles into the blood samples was in a non linear curve. However, the change of the PLT count has been done in each stage of the exposure.

Average absorbed dose via the exposure samples was increased at increasing time of exposure, as shown in Fig.12. This made the number of platelet count decreasing relatively. The reason of this phenomenon due to high dose of alpha particle, this is making a greatest damage to the blood cells. Thus, the alpha particles will lose most of their energy at a short distance in their trajectory for irradiation.

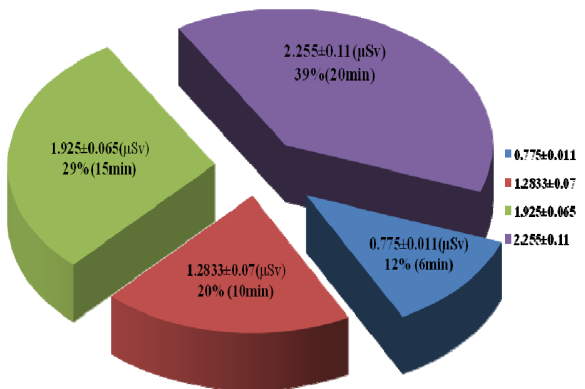

Fig.12: Average absorbed dose increased at increasing time of exposure
As well as, effects of alpha deposition on the surface of blood sample variable, and depended on biological factors, included age and gents. Deposition of alpha particles into the blood samples affected thrombocytopenia (reduction of PLT count) for the cases of male and female in vitro exposure. In addition, the maximum rate of the radon-absorbed dose into the human blood samples was at 20 minutes' time of exposure. This means that the alpha particle density onto the surface of the human blood samples at 20 minutes is more comparing with the other time of direct exposure of alpha particles. Therefore, most the changes of the platelet count of the blood samples were at 20 minutes of exposure for male and female, as shown in Fig.13. Thus, the effect of radon's progenies on thrombocytopenia was high within the highest absorbed dose at 20 minutes. Therefore, the present design considered as a comfortable to make an effect of radon gas on the platelet count of in vitro blood samples.

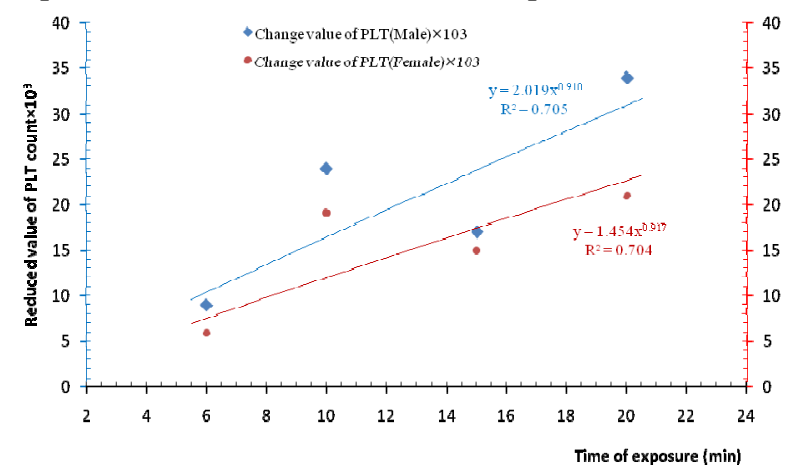

Fig.13: Most change (reduced) of PLT is at 20 minutes exposure of radon gas

On the other hand, deposition of alpha particles into the blood samples affected on an increase of the RBC for the cases of male and female, as shown in Fig.14. We noted that the increase in the time of irradiation leads to increase the number of red blood cells, relatively. However, the percentage changes in the increase in the number of red blood cells were variable according to the type of gender. Maximum change was at the times of 20 and 10 minutes for males and females, respectively. In fact, the changes in platelets were much more compared to what obtained in the number of red blood cells. So we can say that the impact of exposure of blood samples to radon gas is concentrated on the blood platelets.

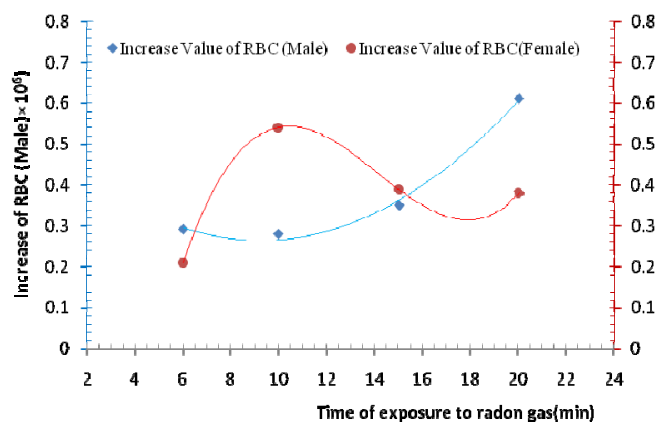

Fig.14: Increase of the RBC and PLT with the time of exposure to radon gas

\section{CONCLUSIONS}

A suitable radon exposure technique had been fabricated to evaluate risks of the accumulation of the radon's progenies 
onto the surface of human blood samples on the concentration of the blood components (PLT, RBC, and WBC). The amount of the loss of concentration $(4.91 \%)$ of radon inside fabricated exposure technique did not affect on its efficiency and applicability to expose of the human blood samples. Assessments of impact radon's progenies deposition on the blood surface showed that the effects of deposition have been affected on reduced concentration of PLT (dieses of thrombocytopenia), and the effects were more on the male blood samples than the females. The changes in PLT were much more compared to what obtained in the number of RBC. The impact of exposure of blood samples to radon gas was concentrated on the PLT. As well as, time of exposure was an important factor to increase or decrease of the effects.

\section{ACKNOWLEDGMENT}

This study was supported by a grant from Universiti Sains Malaysia under account of 1001/PFIZIK/843099.

\section{REFERENCES}

[1] IAEA (International Atomic Energy Agency).Effects of ionizing radiation on blood and blood components: A survey. TECDOC-934.1997.

[2] G. P. Jacobs .A review on the effects of ionizing radiation on blood and blood components. Radiation Physics and Chemistry. Vol. 53. No.5. pp.511-523. 1998.

[3] D. J. Brenner and R. K., Do low dose-rate bystander effects influence domestic radon risks? International Journal of Radiation and Biology. Vol. 78, No.7, pp. 593- 604. 2002.

[4] K. Jefferson. Radiation Worker I Training Study guide. Lab Radiation Control Department. Revision 2.0, pp. 1-73.2010.

[5] N. Joseph and J. Phalen. Online radiology continuing education for radiology professionals. Part 2. 2010.www.SRIM.org
[6] K. Cureton, B. Phillip, H. Patricia , N. Hillary, V. Susan , Z. Linda . Sex difference in maximal oxygen uptake; Effect of equating hemoglobin concentration. Eur J Appl Physiol. Vol. 54, No. 6, pp656-660. 1986.

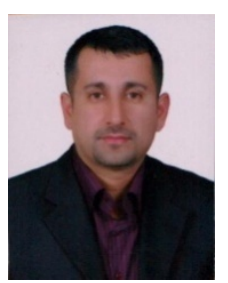

Asaad H. Ismail, Place and date of birth: Erbil-IRAQ, 6 March 1973; Educational background: Participated in more than 15 international conferences and have more that 25 scientific researches in the field of radiation protection dosimetry, especially in risks of radiation on human health/ Medical Physics. He is a lecture in department of physics, University of Salahaddin-Erbil, Iraqi Kurdistan-IRAQ. Member in the international union of radioecology. Temporary, he is working in School of Physics, Universiti Sains Malaysia as a PhD candidate in the field of radiation and Medical Physics. M.Sc degree in Radiation Physics, University of Salahaddin-Erbil, Iraqi Kurdistan-IRAQ. PhD degree in School of Physics, Universiti Sains Malaysia, Penang-Malaysia. Major field is radiation protection dosimetry in Medical and Biophysics.

His email: asadhawlery@hotmail.com,H/P: 0060196890766(In Malaysia) \& 009647504600048 (In Iraq).

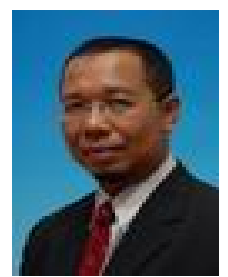

Mohamad S. Jaafar, Chairman of Radiation and Medical Physics Programme, School of Physics, Universiti Sains Malaysia, Penang- MALAYSIA Address: School of Physics, Universiti Sains Malaysia (USM), 11800, Penang-Malaysia (email: msj@usm.my, H/P: 0060194785418)

Research Area: Ultrasound, Instrumentations \& Analytical Science

Radiation Dosimetry, Nuclear Medicine, Medical

Lasers, Monte Carlo Calculations

Academic Coordinator: IAEA PGEC in Radiation Protection and the Safety of Radiation Sources, 2006-present. Chairman, Board of Directors, Koperasi Universiti Sains Malaysia Bhd., 2008-present.

Now he is supervisor for $15 \mathrm{PhD}$ students and more than $15 \mathrm{MSc}$ Students in different fields of Biophysics and medical physics. 